\title{
Frugivoría y dispersión de semillas de la palma Oenocarpus bataua (Arecaceae) en un bosque de los Andes colombianos
}

\author{
Rosario Rojas-Robles ${ }^{1}$, F. Gary Stiles ${ }^{2}$ \& Yaneth Muñoz-Saba ${ }^{2}$ \\ 1. Departamento de Biología, Universidad Nacional de Colombia carrera 45 No 26-85 Bogotá; mrrojasr@unal.edu.co \\ 2. Instituto de Ciencias Naturales, Universidad Nacional de Colombia carrera 45 No 26-85 Bogotá; \\ fgstilesh@unal.edu.co,ydmunozs@unal.edu.co
}

Recibido 31-X-2011. Corregido 20-IV-2012. Aceptado 18-V-2012.

\begin{abstract}
Frugivory and seed dispersal Oenocarpus bataua palm (Arecaceae) in a forest from the Colombian Andes. Seed dispersal is a key process that determines the spatial structure and dynamics of populations of plants, establishes the potential area of recruitment and in this way, the basis for subsequent processes such as predation, germination, competition and growth. The purpose of this research was to identify the guild of frugivores of the Oenocapus bataua palm in fragments of Andean forest, determine the effective dispersers and relate the spatial distribution of palm populations with the dispersion of seeds. To this end, between August 2005-June 2006, observations of the removal of fruits from eight clusters were done, and counting of consumption of fruits beneath 78 palms with mature fruits was undertaken; focal observations of 13 individuals during 90 hours; registration of frugivory with photographic traps during 165 days/nights for a total of 195 photographs and 144 consumption events; experiments with dispersion using 751 perforated fruits/seeds attached to strings and finally, plots to determine spatial distribution all were carried out. In the study area at least five species of mammals ate, dispersed, buried (Sciurus granatensis, Microsciurus mimulus, Dasyprocta punctata y Proechimys sp.), cover (Marmosa robinsoni) and carried the fruits of $O$. bataua off to caves (Marmosa robinsoni y Proechimys sp.) without damaging the seed. A $21.7 \%$ of the fruits were dispersed, 13.2 gnawed or peeled, 5.6\% covered, buried and carried to caves. The average distance of removal of seeds and fruits was $3.1 \mathrm{~m}$ although in a lesser proportion, dispersion events of $>50 \mathrm{~m}$ were recorded. The abundant production of fruits, their size and weight, their intense removal by frugivores, the short dispersion distances, the absence of large size frugivores (reduced by hunting and fragmentation), that might perform long-distance dispersion, and the increase of rodents, especially squirrels, that strongly pressure the fruit resource, generate a spatially restricted seed rain of seeds responsible for the aggregate patterns of distribution of seeds, seedlings and subsequently of juveniles and sub-adults. However the short time and high percentage of seed germination and the robustness of seeds and seedlings give $O$. bataua the capacity to survive in dense aggregations. Therefore, despite the limitations on dispersion, this is a dominant species in the study area. Populations of $O$. bataua have been reduced by intense exploitation and by the transformation and reduction of their natural habitat. The over-exploitation of the fruits or the juvenile phases of this palm can affect the age structure and population dynamics. Therefore, the management and exploitation of this palm in the future will depend to large degree on the conservation of forest habitats. Rev. Biol. Trop. 60 (4): 1445-1461. Epub 2012 December 01.
\end{abstract}

Key words: Oenocarpus bataua, palms, frugivory, camera trapping, spatial distribution, fragmentation, defaunation, Colombia.

La dispersión de semillas se considera un proceso clave que determina la estructura espacial y la dinámica de las poblaciones de plantas (Nathan \& Muller-Landau 2000); establece el área potencial de reclutamiento y de esta forma, la base para procesos subsecuentes tales como depredación, germinación, competencia y crecimiento (Barot et al. 1999, Nathan \& MullerLandau 2000, Wang \& Smith 2002).

La dispersión de semillas mueve la descendencia lejos de la influencia de factores de mortalidad denso-dependientes, particularmente en 
la vecindad del adulto parental (Connell 1971, Janzen 1970). Puede permitir la colonización de microhábitats donde la probabilidad de establecimiento y supervivencia sean mayores (Hamilton \& May 1977, Howe \& Smallwood 1982). La dispersión ocurre por el efecto combinado de un gremio de consumidores y estos difieren en la contribución que hacen a la reproducción futura de la planta, mediante la dispersión de semillas (Hollander \& Vander Wall 2004), la supervivencia de semillas y el reclutamiento de las plántulas (Howe 1990, Jordano 2000, Jordano \& Schupp 2000, Jordano \& Godoy 2002). Determinar cómo difieren las especies en su eficiencia como dispersores es importante para comprender la evolución de esta interacción mutualista (Howe 1977, Snow 1981).

Los primeros estudios en dispersión de semillas no lograron proveer un conteo completo de los diversos destinos y varias direcciones que una población de semillas podía seguir entre producción y germinación (Vander Wall et al. 2005). Una aproximación integral de la dispersión de semillas, que cubra los estadios de dispersión y post-dispersión (dispersión secundaria), abordados desde una perspectiva de efectividad de los dispersores, es una importante herramienta para desarrollar un entendimiento de las consecuencias ecológicas y evolutivas de mutualismos entre plantas y frugívoros (Jordano \& Schupp 2000).

La modificación de los hábitats por fragmentación o por cacería reduce los vertebrados frugívoros y herbívoros, produciendo cambios en los patrones espaciales de la regeneración y en la diversidad de las especies (Dirzo \& Miranda 1990, Cordeiro \& Howe 2003). Alteraciones en la composición de la fauna, particularmente de grandes depredadores, pueden llevar a modificaciones en las interacciones entre las plantas y los animales frugívoros que consumen, depredan y dispersan sus semillas (Jordano et al. 2006). Cambios en la estructura de la comunidad de frugívoros, en los cuales las especies pequeñas y generalistas son favorecidas en detrimento de especies grandes, producen serias implicaciones en la dispersión y depredación de semillas (Fleury \& Galetti 2006) y a mediano y largo plazo en la distribución espacial de las especies vegetales (Silva \& Tabarelli 2001).

Las palmas constituyen uno de los componentes más abundantes y característicos de los bosques tropicales húmedos (Kahn \& de Granville 1992). Sus flores y frutos son recursos alimenticios importantes para muchos animales (Zona \& Henderson 1989, Henderson 2002), y algunas palmas se han considerado especies clave, pues sus frutos son importantes para los frugívoros durante períodos de escasez (Peres 1994). Una de estas especies es la palma milpesos (Oenocarpus bataua Mart.), sobre la cual se ha documentado, que sus frutos son consumidos por diversas especies de vertebrados, en zonas bajas tropicales, en bosques continuos y relativamente poco intervenidos (Zona \& Herderson 1989, Sist 1989, Bodmer 1991, Kahn \& Granville 1992, Peres 1994, Stevenson et al. 2000). Sin embargo, no hay estudios de dispersión de más largo plazo con esta especie.

Esta constituye la primera investigación sobre la ecología de la dispersión de semillas de $O$. bataua en un bosque subandino. El objetivo de este trabajo fue identificar el gremio de frugívoros en el área de estudio y determinar cuáles son los dispersores efectivos en términos de número de semillas dispersadas y calidad, en términos de tratamiento dado a las semillas, distancias y destinos a los que éstas son llevadas; de igual forma, discutir cómo la distribución espacial de semillas, plántulas, juveniles y adultos observada en el área de estudio en trabajos preliminares (Rojas-Robles 2008, Rojas-Robles et al. 2008), puede estar relacionada con las interacciones faunísticas mediante la dispersión de semillas. Entender procesos ecológicos fundamentales, como la dispersión de semillas y los posibles cambios en estos procesos por defaunación y fragmentación, puede ser importante para el manejo y la conservación de las poblaciones de O.bataua que es una especie ampliamente utilizada y explotada en todo su ámbito de distribución. 


\section{MATERIALES Y MÉTODOS}

Área de estudio: La investigación se llevó a cabo en un área de bosque subandino o bosque húmedo premontano (bh-PM) (Holdridge 1996), en la cuenca media del río Porce $\left(6^{\circ} 45^{\prime} 37^{\prime \prime} \mathrm{N}-75^{\circ} 06^{\prime} 28^{\prime \prime} \mathrm{W}\right)$, sobre la Cordillera Central andina de Colombia. Los sitios de muestreo fueron tres fragmentos de bosque primario localizados entre $925-1100 \mathrm{~m}$ de altitud: (a) Normandía, un fragmento continuo de 268ha, (b) La Piedra con 27.9ha, separado del anterior por una carretera y (c) San Ignacio con un área de 71.3ha, aislado y de difícil acceso a $10 \mathrm{~km}$ de los dos anteriores rodeado de pastizales y algunos parches de bosque. Estos fragmentos pertenecen a Empresas Públicas de Medellín (EPM) y están relativamente protegidos. Los dos primeros fragmentos se encuentran sobre la carretera principal y a pesar de que hay guardabosques, los pobladores entran a cazar, extraer hojas de $O$. bataua y madera.

En los fragmentos estudiados se han registrado 237 especies de aves (Corporación Ambiental 2001), entre las cuales se destacan: Aburria aburri, Ortalis columbiana, Pteroglossus torquatus, Ramphastus citreolaemus, Ramphastus swainsonii, Steatornis caripensis y Tinamus major como posibles consumidores de los frutos de O. bataua. En el área de estudio se encuentran los primates: Alouatta seniculus, Saguinus leucopus y Aotus lemurinus. Del orden Carnivora están las especies: Nasua nasua, Potos flavus, Cerdocyon thous, Procyon cancrivorus y Leopardus pardalis. Roedores grandes como la paca (Cuniculus paca) y el Aguti (Dasyprocta punctata) son intensamente cazados en los fragmentos de bosque en los que aún están presentes. Ya no existen en la zona el tapir (Tapirus bairdii), pecaríes (Pecari tajacu y Tayassu pecari) ni el ciervo (Mazama americana).

Especie estudiada: La palma Oenocarpus bataua (Arecaceae) localmente conocida como milpesos se encuentra en toda la región tropical de Suramérica (Galeano \& Bernal 1987, Balick 1992, Henderson et al. 1995), hasta los $1400 \mathrm{~m}$ de elevación (Borchsenius et al. 1998). Es ampliamente utilizada y promisoria. Es una palma arborescente con alturas entre 4-26m, es un elemento dominante del dosel y el sub-dosel. Es monoica, con inflorescencias de hasta dos metros de largo. Los frutos tienen $35.3 \pm 2.8 \mathrm{~mm}$ de largo, $21.3 \pm 2 \mathrm{~mm}$ de diámetro, $10.9 \pm 2.1 \mathrm{~g}$ de peso (media $\pm 1 \mathrm{DS} \mathrm{n}=100$ ), que sirven como alimento a la fauna silvestre frugívora. En el área de estudio encontramos un valor medio de 1366 frutos por racimo $(n=17)$.

Observaciones focales de frugivoría: Entre septiembre 2005-septiembre 2006 y en la medida que se encontraban palmas con frutos maduros, se realizaron observaciones focales entre 6-10 de la mañana, con binoculares (Bushnell ${ }^{\circledR}$ Image View ${ }^{\circledR}$ ) dentro de una carpa camuflada. Se realizaron 38 jornadas, para un total de 90 horas de observación en 13 palmas. A los frugívoros visitantes se les registró hora de llegada, tiempo de permanencia, tiempo consumiendo, número de frutos consumidos, llevados y derribados (Jordano \& Schup 2000).

Remoción de frutos en el racimo: En diferentes meses y palmas y en la medida que hubo racimos de frutos verdes, en ocho de ellos marcamos y numeramos con cinta reflectiva 470 raquilas, a las cuales se les contó el número de frutos, en total 3 646. Cada mes se contó el número de frutos en las raquilas, hasta que el racimo maduro quedó sin frutos por la remoción de los frugívoros.

Consumo de frutos debajo de palmas con racimos maduros: De mayo 2003-mayo 2007 hicimos seguimiento mensual a la fenología reproductiva de O. bataua (Rojas \& Stiles 2009), especialmente la presencia de frutos maduros en 104 palmas. Adicionalmente, realizamos observaciones de frugivoría, contando los frutos roídos, pelados o partidos que se encontraron debajo de las palmas y en un radio de $5 \mathrm{~m}$ alrededor de las mismas, en los fragmentos de Normandía y la Piedra. Por el tamaño, tipo de marcas (dientes) y patrón de la 
mordida dejada en los frutos, determinamos los posibles frugívoros.

\section{Registro de frugivoría con trampas foto-} gráficas: Con el fin de identificar frugívoros diurnos, nocturnos y aquellos poco conspicuos, entre agosto 2005-junio 2006 colocamos dos trampas fotográficas tipo "Camtrakker" o "Camera Trapping", con sensor de movimiento y calor que se dispara cuando entra un objeto en su campo de detección. Dejamos las cámaras activadas de forma continua (día y noche), apuntando a racimos maduros en la palma a alturas entre $7-17 \mathrm{~m}$. Igualmente colocamos las cámaras apuntando a montones de frutos en el suelo, en palmas con frutos maduros cayendo o con evidencias de que previamente estaban siendo visitadas por los frugívoros. Colocamos las cámaras haciendo un esfuerzo de muestreo semejante (días/cámara) tanto en los tres fragmentos de bosque, como en el suelo y en los racimos.

Dispersión de frutos y semillas: Entre agosto 2005-diciembre 2006 recolectamos y perforamos 751 frutos y semillas. Cada fruto o semilla fue atravesado con alambre de pesca y a este amarramos un hilo resistente de $50 \mathrm{~cm}$ de largo, al final del cual colocamos una cinta reflectiva (frutos y semillas móviles). En otros un carretel con hilo, que dejamos amarrado a la base de un arbusto, permitiendo que el hilo saliera libremente (carretes fijos) (Forget \& Wenny 2005). Para la cantidad de frutos y semillas de cada método y fragmento ver cuadro 5. Los frutos y semillas se colocaron en los tres fragmentos de bosque a una distancia de $5 \mathrm{~m}$ entre ellos. Después de cuatro a seis días revisamos para ver si habían sido pelados, roídos o partidos y si habían sido dispersados o no. También medimos las distancias de remoción y los destinos a los que fueron llevados. Registramos si habían sido tapados, enterrados o llevados a cuevas (Silva \& Tabarelli 2001).

Distribución espacial de semillas y plántulas: Para determinar patrones de distribución de semillas y plántulas en cada fragmento, subdividimos un área de una hectárea en 100 cuadrantes de $10 \times 10 \mathrm{~m}$, y en el vértice de cada cuadrante hicimos sub-parcelas de $1 \mathrm{~m}^{2}$ para un total de 100 sub-parcelas en cada fragmento. Estos vértices se hicieron trazando el cuadrante y tomando la medida en el punto exacto, sin hacer parcelas ni cuadrículas, pues al hacerlo se altera el área pisoteando semillas y plántulas. En cada sub-parcela se contó el número de semillas y plántulas (hasta $40 \mathrm{~cm}$ y una hoja) y se midió la distancia del centro del cuadrante al vecino reproductivo más cercano, definido como la palma más cercana mostrando eventos reproductivos como racimos, racimos secos en la palma o en el suelo.

Utilizamos la prueba de chi-cuadrado para: comparar frugivoría en el suelo y en el racimo; diferencias en la dispersión de frutos con relación a semillas; diferencias entre semillas y frutos tapados, enterrado y llevado a las cuevas y para comparar dispersión de semillas y frutos entre los fragmentos de bosque muestreados. Se utilizó la correlación de Spearman $\left(\mathrm{r}_{\mathrm{s}}\right)$ para relacionar el número de noches que se dejaron los frutos y el número de semillas y frutos dispersados. Para comparar las distribuciones de frecuencias de las distancias de remoción de semillas y frutos y para comparar las distancias de semillas y frutos enterrados, tapados y llevados a las cuevas se uso la prueba de Kolmogorov-Smirnov. Como los valores de las distancias de semillas y plántulas al vecino reproductivo más cercano no presentaron una distribución normal, ni al realizar transformación logarítmica, se uso la prueba no paramétrica de Kruskal-Wallis (H) para comparar el comportamiento de las distancias al vecino más cercano en los fragmentos muestreados.

\section{RESULTADOS}

Observaciones focales: La especie de frugívoro más importante en términos de número y tiempo de visitas, así como por el número de frutos manipulados y consumidos fue la ardilla Microsciurus mimulus, seguida por la ardilla Sciurus granatensis (Cuadro 1). La presencia de aves fue muy rara, solamente se observó en 
CUADRO 1

Número y tiempo de visita de frugívoros a racimos maduros de O. bataua de agosto 2005-septiembre 2006

TABLE 1

Number and time of visit of frugivores, to ripe clusters of O. bataua from August 2005-September 2006

\begin{tabular}{|c|c|c|c|c|c|c|}
\hline Especie & $\begin{array}{l}\text { N. }{ }^{\circ} \text { de } \\
\text { visitas }\end{array}$ & $\begin{array}{c}\text { Tiempo total } \\
\text { de visitas }\end{array}$ & $\begin{array}{l}\text { Frutos } \\
\text { llevados }\end{array}$ & $\begin{array}{c}\text { Frutos } \\
\text { consumidos }\end{array}$ & $\begin{array}{l}\text { Frutos } \\
\text { tumbados }\end{array}$ & $\begin{array}{c}\text { Palmas } \\
\text { visitadas }\end{array}$ \\
\hline \multicolumn{7}{|l|}{ Aves } \\
\hline Cyanocorax affinis & 7 & 3'56' & 3 & 0 & 0 & 2 \\
\hline \multicolumn{7}{|l|}{ Mamíferos } \\
\hline Microsciurus mimulus & 68 & $155^{\prime} 56^{\prime \prime}$ & 62 & 12 & 18 & 7 \\
\hline Sciurus granatensis & 22 & $75^{\prime} 15^{\prime \prime}$ & 30 & 40 & 47 & 4 \\
\hline Totales & 97 & $235^{\prime} 07^{\prime \prime}$ & 95 & 52 & 65 & \\
\hline
\end{tabular}

1. Tiempo en minutos y segundos.

pocas ocasiones y por muy poco tiempo a la urraca Cyanocorax affinis. Las ardillas llevan el fruto a una percha a unos $4-10 \mathrm{~m}$ del racimo, consumen el mesocarpo del fruto, dejan caer semillas enteras al suelo y regresan nuevamente por otro fruto, repitiendo varias veces este comportamiento hasta ser interrumpidas, en ocasiones por otras ardillas. También acostumbran cortar la raquila y llevarse de dos a cuatro frutos lejos de la palma, en una ocasión hasta $63 \mathrm{~m}$. Generalmente, las raquilas con frutos maduros cortadas por ardillas con frutos maduros caen al suelo y quedan disponibles para otros frugívoros y posibles dispersores secundarios.

Remoción de frutos en los racimos: En las raquilas marcadas la remoción de los frutos fue del $100 \%$ en un tiempo de $58 \pm 22$ días (Coeficiente de Variación=38\%). Por las observaciones focales se determinó que el $28.7 \%$ $( \pm 30.8 \%$, C.V. $=107)$ de las raquilas con frutos maduros marcadas, fueron cortadas por las ardillas; sólo el $0.61 \%$ de raquilas fueron cortadas cuando los racimos aún tenían los frutos verdes, lo que deja ver que la depredación predispersión es baja. Las ardillas cortan las raquilas posiblemente para cargar mayor número de frutos y transportarlos más fácilmente; muchas veces dejan caer los frutos y los consumen en el suelo.

Consumo de frutos caídos debajo de palmas con racimos maduros: Aunque las observaciones fenológicas se hicieron entre mayo 2003-mayo 2007, la producción de frutos maduros en más del $10 \%$ de las palmas, se presentó entre marzo 2004-agosto 2005 (Rojas \& Stiles 2009). Los principales picos de consumo coincidieron con el periodo de mayor producción de frutos (Fig. 1).

De acuerdo con el patrón de la mordida en los frutos, observaciones focales de frugivoría y registros fotográficos en los tres fragmentos, las ardillas ( $S$. granatensis y M. mimulus), el agutí (Dasyprocta punctata) y la rata espinosa (Proechimys sp.) fueron los frugívoros que más consumieron el mesocarpo de los frutos que contienen sustancias ricas en grasas y proteínas (Cuadro 2). Los frugívoros no dañaron las semillas que son de consistencia bastante dura y fibrosa y no contienen nutrientes, como para ser dañadas por mamíferos pequeños y medianos. Solo son partidas por los fuertes dientes de Cuniculus paca, que mostró el menor porcentaje de consumo de frutos y en la mayoría de los casos rompió la semilla. En los fragmentos de Normandía y La Piedra, en orden de importancia las especies que más consumieron los frutos fueron las mismas (Cuadro 2).

Registro de frugivoría con trampas fotográficas: De 165 días y noches de trabajo efectivo de las cámaras, sólo en 70 días/noches se tomaron 231 fotos de fauna vertebrada: 36 de aves en las cuales no hubo registro de consumo de frutos y 195 de mamíferos, en las que se 


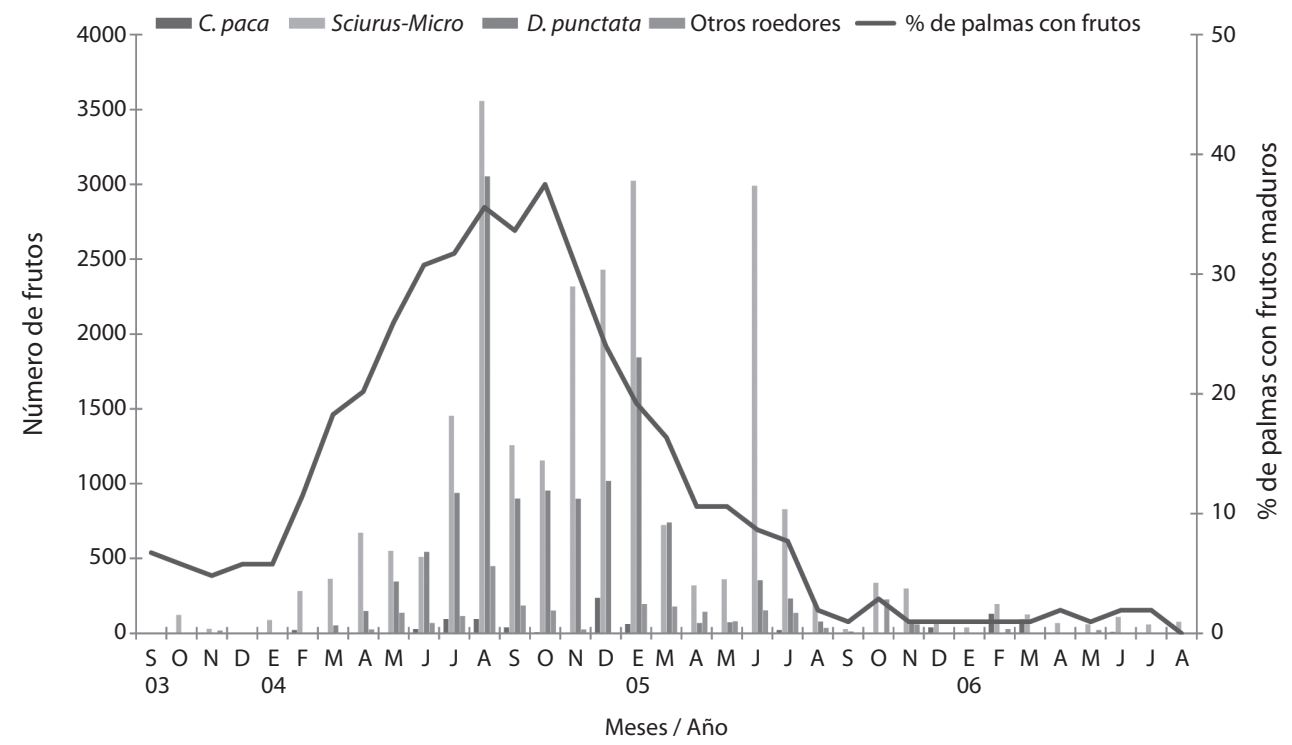

Fig. 1. Número de frutos roídos o pelados por frugívoros encontrados y contados bajo palmas de $O$. bataua y porcentaje de palmas con frutos maduros de septiembre 2003-agosto 2006.

Fig. 1. Number of fruits gnawed or peeled by frugivores found and counted under palms of $O$. bataua and percentage of palms with ripe fruits September 2003-August 2006.

\section{CUADRO 2}

Porcentaje de frutos roídos y pelados por frugívoros en palmas de $O$. bataua a las que se les dio seguimiento de mayo 2003-mayo 2007

TABLE 2

Percentage of fruits gnawed or peeled by frugivores under palms of O. bataua from May 2003-May 2007

\begin{tabular}{lcccccccc}
\multicolumn{1}{c}{ Fragmento } & Registros & Palmas $^{1}$ & \multicolumn{7}{c}{ \% de frutos roídos por frugívoros } \\
Normandía & 243 & $53 / 78$ & 71.23 & 19.09 & 6.43 & 2.98 & 0.27 & 26646 \\
La Piedra & 212 & $23 / 26$ & 55.37 & 36.08 & 6.43 & 1.97 & 0.15 & 24140 \\
Totales $^{2}$ & 455 & $76 / 104$ & 63.69 & 27.16 & 6.43 & 2.50 & 0.22 & 50786 \\
\hline
\end{tabular}

1. Palmas con frutos maduros/palmas totales marcadas en cada fragmento.

2. No se hizo seguimiento de frugivoría en el fragmento de San Ignacio.

3. Las especies de ardillas fueron Sciurus granatensis y Microsciurus mimulus

4. N.I. No identificada la especie de frugívoro.

1. Palms with ripe fruits/total palms marked in every fragment.

2. No followed frugivory in the fragment of San Ignacio.

3. The squirrel species are Sciurus granatensis and Microsciurus mimulus

4. N.I. Frugivores species unidentified. 
fotografiaron siete especies y 144 registros de consumo de frutos. Cinco especies incluyeron en su dieta frutos de $O$. bataua. La mayor frecuencia de consumo fue de la ardilla $S$. granatensis, seguida por la rata espinosa del género Proechimys sp., y la ardilla M. mimulus. También fueron fotografiados consumiendo frutos, el agutí Dasyprocta punctata, y el marsupial Marmosa robinsoni (Cuadro 3).

Se registró mayor consumo de frutos de $O$. bataua por mamíferos en los racimos que en el suelo $\left(\chi^{2}, p<0.005\right)$; no obstante, el gremio de frugívoros en el suelo es más diverso, cuatro especies contra dos en el racimo (Cuadro 3). Las ardillas $S$. granatensis y $M$. mimulus fueron las únicas especies fotografiadas consumiendo en el racimo. S. granatensis también fue registrada y observada consumiendo en el suelo.

El mayor número de registros de frugivoría se obtuvo entre octubre-noviembre 2005, periodo incluido dentro de aquel con mayor actividad de frugivoría en las palmas a las que se les hizo seguimiento fenológico. La rata espinosa Proechimys sp. fue la especie más común en el mayor número de meses. Al comparar registros de consumo de frutos en los fragmentos, notamos que San Ignacio tuvo el menor número de registros, pero el mayor número de especies fotografiadas, aunque no todas consumiendo (Cuadro 4).

Las trampas fotográficas fueron especialmente útiles para registrar especies frugívoras nocturnas y otras de difícil observación e inconspicuas, que no fueron registradas en las observaciones focales, ya que los focales se hicieron en las horas de mayor actividad de los frugívoros diurnos. Igualmente, fueron una importante herramienta para determinar el gremio de frugívoros, pues la frecuencia diferencial de registros de consumo, nos ayuda a determinar las especies frugívoras más importantes para la palma.

Pudimos establecer diferencias en los estratos, en las horas del día y en los meses del año, en los que cada especie consume los frutos. De las siete especies registradas, cinco incluyeron en su dieta frutos de O. bataua. El $74 \%$ de los registros fotográficos fueron de consumo (Cuadro 4), lo que muestra gran actividad de los frugívoros. Las especies más importantes en el consumo de frutos en términos de número de visitas, frecuencia de consumo y presencia consumiendo en varios meses del año son en orden: $S$. granatensis, $M$. mimulus y Proechimys sp. Las dos primeras especies también fueron las más importantes

\section{CUADRO 3}

Número de fotografías tomadas, frecuencia de registros por especie, hábito de los frugívoros, estrato de forrajeo de frutos de la palma O. bataua de agosto 2005-junio 2006

TABLE 3

Number of pictures taken, frequency record by species and stratum of foraging on the O. bataua palm from August 2005-June 2006

\begin{tabular}{lccccc}
\multicolumn{1}{c}{ Especies } & $\begin{array}{c}\text { N. }{ }^{\circ} \text { de registro } \\
\text { fotográficos }\end{array}$ & Estrato & Fragmentos & Hábito & $\begin{array}{c}\text { Frecuencia de } \\
\text { registro de consumo }\end{array}$ \\
Roedor (no identificado) & 1 & $\mathrm{~S}$ & $\mathrm{I}$ & No & - \\
Leopardus pardalis & 1 & $\mathrm{~S}$ & $\mathrm{I}$ & No & - \\
Proechimys sp. $(350)^{*}$ & 44 & $\mathrm{~S}$ & $\mathrm{I}-\mathrm{P}-\mathrm{N}$ & $\mathrm{No}$ & 0.20 \\
Marmosa robinsoni $(84)^{*}$ & 19 & $\mathrm{~S}$ & $\mathrm{I}$ & $\mathrm{No}$ & 0.06 \\
Dasyprocta punctata $(3 \mathrm{700})^{*}$ & 13 & $\mathrm{~S}$ & $\mathrm{I}-\mathrm{P}-\mathrm{N}$ & $\mathrm{D}$ & 0.05 \\
Microsciurus mimulus $(120)^{*}$ & 42 & $\mathrm{R}$ & $\mathrm{P}-\mathrm{N}$ & $\mathrm{D}$ & 0.28 \\
Sciurus granatensis $(366)^{*}$ & 75 & $\mathrm{R} / \mathrm{S}$ & $\mathrm{I}-\mathrm{P}-\mathrm{N}$ & $\mathrm{D}$ & 0.41 \\
Total & 195 & & & & \\
\hline
\end{tabular}

* Peso medio en gramos. Datos tomados de Emmons \& Feer (1999).

$\mathrm{S}=$ suelo; $\mathrm{R}=$ racimo; $\mathrm{I}=$ San Ignacio; $\mathrm{P}=$ La Piedra; $\mathrm{N}$ = Normandía; $\mathrm{D}=$ Diurno; No = Nocturno. 
CUADRO 4

Registros fotográficos de mamíferos que consumen la palma $O$. bataua teniendo en cuenta fragmento, estrato, porcentaje de registro de frugivoría, agosto 2005-junio 2006

TABLE 4

Photographic records of mammals frugivores in O. bataua in the fragments from August 2005-June 2006

\begin{tabular}{lcccc}
\multicolumn{1}{r}{ Fragmento } & Días/Cámara & N. $^{\circ}$ de fotos & Eventos de consumo (\%) & N. $^{\circ}$ Especies consumiendo \\
Normandía & 52 & 56 & $33(59 \%)$ & $4 / 4$ \\
La Piedra & 71 & 105 & $94(89 \%)$ & $4 / 4$ \\
San Ignacio & 42 & 34 & $17(50 \%)$ & $3 / 6$ \\
Total Suelo & 82 & 82 & $47(57 \%)$ & $4 / 6$ \\
Total Racimo & 83 & 113 & $97(86 \%)$ & $2 / 2$ \\
Total & 165 & 195 & $144(74 \%)$ & \\
\hline
\end{tabular}

en las observaciones focales. El fragmento de La Piedra mostró mayor porcentaje de registros de consumo $(\chi 2 \mathrm{p}<0.005)$ que Normandía y San Ignacio.

Dispersión de frutos y semillas: De los 751 frutos y semillas colocados, $21.7 \%$ fueron dispersados, $13.2 \%$ fueron roídos o pelados, $5.6 \%$ fueron tapados, enterrados y llevados a cuevas (Cuadro 5). Mayor número de frutos fueron dispersados en comparación con las semillas $\left(\chi^{2}, \mathrm{p}>0.005\right)$. Esto se explica por el hecho de que son más apetecidos los frutos, pues es el mesocarpo el que consumen. Por otra parte, no hubo diferencias significativas entre el número de semillas y frutos tapados, enterrados y llevados a las cuevas.

Hubo correlación positiva entre el tiempo (número de noches-días) que se dejaron los frutos expuestos y el número de frutos y semillas dispersados por los frugívoros (Spearman, $\mathrm{p}<0.05)$. Al relacionar sólo semillas dispersadas (Spearman, $\mathrm{p}>0.05$ ), no fue significativa la correlación, pero al correlacionar solo frutos dispersados (Spearman, $\mathrm{p}<0.05$ ), nuevamente la relación fue significativa. De esta forma, podríamos sugerir que frutos y en menor medida semillas que permanezcan más tiempo expuestos, tienen mayor posibilidad de ser encontrados y dispersadas por los frugívoros.

Los fragmentos de San Ignacio y La Piedra presentaron mayor número de frutos y semillas dispersados $\left(\chi^{2}, \mathrm{p}<0.05\right)$ que el fragmento de Normandía (Cuadro 6).

La distancia media de remoción de semillas y frutos fue $3.1 \mathrm{~m}(\mathrm{DE}=5.46, \mathrm{n}=140$, $\mathrm{CV}=176 \%$ ). La distancia mínima fue de $0.1 \mathrm{~m}$ y la distancia máxima de remoción fue $53 \mathrm{~m}$. La mayoría de las distancias no sobrepasaron los 14m (Fig. 2). Los frutos fueron dispersados a mayores distancias que las semillas (Kolmogorov-Smirnov, $\mathrm{p}<0.005$ ). Los frutos llevados a las cuevas fueron dispersados a mayor distancia que los enterrados y tapados (KolmogorovSmirnov, $\mathrm{p}<0.05$ ) (Fig. 2).

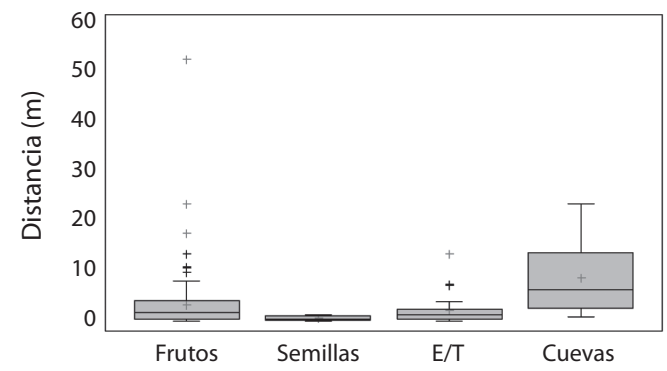

Fig. 2. Diagrama de cajas de las distancias de dispersión de semilla y frutos de $O$. bataua removidos, enterrados y tapados $(\mathrm{E} / \mathrm{T})$ y llevados a cuevas por frugívoros, agosto 2005-diciembre 2006.

Fig. 2. Box-plot of the distances of dispersal of seeds and fruits of $O$. bataua removed, buried and covered (E/T) and carried to caves by frugivores, August 2005 to December 2006. 
CUADRO 5

Semillas y frutos de O. bataua dispersados por mamíferos, agosto 2005-diciembre 2006

TABLE 5

Seeds and fruits of O. bataua dispersed by mammals, August 2005-December 2006

\begin{tabular}{|c|c|c|c|c|c|c|c|c|}
\hline Área & Colocados & $\begin{array}{c}\text { No } \\
\text { Dispersado }\end{array}$ & Dispersado & $\begin{array}{c}\text { No } \\
\text { hallado }^{1}\end{array}$ & $\begin{array}{l}\text { Roído/ } \\
\text { pelado }\end{array}$ & Tapado & Enterrado & En cueva \\
\hline \multicolumn{9}{|l|}{ Semillas fijas } \\
\hline Normandía & 60 & 57 & 2 & 0 & - & 1 & 1 & 0 \\
\hline La Piedra & 50 & 41 & 9 & 0 & - & 1 & 2 & 1 \\
\hline San Ignacio & 20 & 14 & 6 & 0 & - & 0 & 1 & 0 \\
\hline Sub-totales & 130 & 112 & 17 & 0 & - & 2 & 4 & 1 \\
\hline \multicolumn{9}{|l|}{ Semillas móviles } \\
\hline Normandía & 75 & 74 & 1 & 0 & - & 0 & 0 & 0 \\
\hline \multicolumn{9}{|l|}{ Frutos móviles } \\
\hline La Piedra & 71 & 52 & 19 & 6 & 13 & 1 & 3 & 0 \\
\hline Normandía & 106 & 87 & 19 & 8 & 10 & 3 & 3 & 2 \\
\hline San Ignacio & 40 & 37 & 3 & 0 & 5 & 0 & 0 & 0 \\
\hline Sub-total & 217 & 176 & 41 & 14 & 28 & 4 & 6 & 2 \\
\hline Frutos fijos & & & & & 0 & & & \\
\hline La Piedra & 100 & 67 & 33 & 0 & 26 & 4 & 4 & 1 \\
\hline Normandía & 129 & 91 & 38 & 3 & 29 & 6 & 1 & 4 \\
\hline San Ignacio & 100 & 67 & 33 & 1 & 16 & 1 & 2 & 0 \\
\hline Sub-total & 329 & 225 & 104 & 4 & 71 & 11 & 7 & 5 \\
\hline Semillas Totales & 205 & 186 & 18 & 0 & - & 2 & 4 & 1 \\
\hline Frutos Totales & 546 & 401 & 145 & 18 & 99 & 15 & 13 & 7 \\
\hline Total General & 751 & ${ }^{2} 587$ & 163 & 18 & 99 & 17 & 17 & 8 \\
\hline Porcentaje & 100 & 78.16 & 21.70 & 2.40 & 13.18 & 2.26 & 2.26 & 1.07 \\
\hline
\end{tabular}

1. Estos frutos fueron dispersados no hallados.

2. De los 751 frutos y semillas solo un carretel se perdió, no hallamos el lugar donde se había dejado originalmente.

\section{CUADRO 6}

Semillas y frutos de $O$. bataua dispersados, roídos, enterrados, tapados y llevados a cuevas en los tres fragmentos de bosque estudiados de agosto2005-diciembre 2006

TABLE 6

Seeds and fruits of $O$. bataua scattered, gnawed, buried and carried caves in three forest fragments studied from August 2005-December 2006

\begin{tabular}{lccccc}
\multicolumn{1}{c}{ Área } & Colocados & Dispersados (\%) & Roídos/pelados (\%) & ${ }^{2}$ T/E (\%) & En cuevas (\%) \\
Normandía & 370 & $16.2^{1}(60)$ & $10.5(39)$ & $3.5(13)$ & $1.62(6)$ \\
San Ignacio & 160 & $26.2(42)$ & $13.1(21)$ & $2.5(4)$ & 0 \\
La Piedra & 221 & $27.6(61)$ & $17.6(39)$ & $6.8(15)$ & $0.9(2)$ \\
\hline
\end{tabular}

1. Porcentaje de semillas y frutos (número).

2. Frutos y semillas tapados y enterrados. 
En el área de estudio demostramos que al menos cinco especies de mamíferos comen, dispersan o, entierran frutos ( $S$. granatensis, D. punctata y Proechimys sp.), tapan ( $M$. robinsoni) y llevan a las cuevas (M. robinsoni y Proechimys sp.), los frutos de O. bataua, en la mayoría de los casos sin dañar la semilla. Debido a que al perforar la semilla para colocarle el alambre de pesca el embrión se daña, no pudimos obtener datos de germinación de las semillas dispersadas en estos experimentos.

\section{Distribución espacial de semillas y plán-} tulas: Normandía mostró el mayor número de parcelas de un metro cuadrado con semillas, pero en total la menor cantidad de semillas. $\mathrm{La}$ Piedra presentó el mayor número de semillas y plántulas (Cuadro 7).

En promedio se hallaron 163 semillas y 155 plántulas en $100 \mathrm{~m}^{2}$. La producción de frutos en el área de estudio no es continua en el tiempo, lo cual podría permitir distinguir «cohortes de semillas». Adicionalmente, las parcelas se hicieron entre diciembre 2005mayo 2006, cuando el pico de producción de frutos maduros había finalizado (Rojas \& Stiles 2009) y ya los frutos habían caído. Esto podría explicar el alto número de semillas y el hecho de que muchas estuvieran ya muertas al hacer el conteo en las parcelas. Sin embargo, dado el ciclo supra-anual de producción de semillas, este dato podría variar mucho en el tiempo.

San Ignacio mostró las mayores distancias medias al vecino reproductivo más cercano, tanto para semillas como para plántulas. Existe una amplia variación en las distancias de semillas y plántulas al vecino reproductivo más cercano, representada en los altos valores del coeficiente de variación (Cuadro 7). Se observó una correlación negativa entre el número de semillas (Spearman, $\mathrm{p}<0.005$ ) y de plántulas (Spearman, $\mathrm{p}<0.05$ ) con la distancia al vecino reproductivo más cercano. Como era de esperarse, a mayor distancia de la palma parental menor número de semillas y plántulas.

Las distancias de semillas al vecino reproductivo más cercano estuvieron entre $0-50 \mathrm{~m}$ pero la mayoría estuvo entre los $0-15 \mathrm{~m}$ (Fig. 3a), comportamiento muy parecido al de las plántulas (Fig. 3b). Los valores extremos en las distancias evidencian eventos de dispersión de semillas por frugívoros, debido a que por el peso estas semillas no son dispersadas por viento, en el área no son trasportadas por agua y en algunos casos el vecino reproductivo se encontró pendiente abajo.

CUADRO 7

Media, desviación estándar y coeficiente de variación de las distancias de semillas y plántulas de $O$. bataua al vecino reproductivo más cercano en los tres fragmentos de bosque estudiados

TABLE 7

Mean, standard deviation and coefficient of variation to the distance of seed and seedlings to $O$. bataua to the reproductive nearest neighbor in three forest fragments studied

\begin{tabular}{|c|c|c|c|}
\hline Fragmento & $\begin{array}{l}\text { N. }{ }^{\circ} \text { de semillas o plántulas } \\
\text { (N. }{ }^{o} \text { parcelas con semillas o plántulas) }\end{array}$ & $\begin{array}{l}\text { Distancia al vecino } \\
\text { reproductivo Media } \pm \text { DS }\end{array}$ & $\begin{array}{l}\text { Coeficiente de } \\
\text { Variación }(\%)\end{array}$ \\
\hline \multicolumn{4}{|l|}{ Semillas } \\
\hline Normandía & $117(51)$ & $8.32 \pm 9.19$ & 110.47 \\
\hline La Piedra & 197 (22) & $7.87 \pm 5.94$ & 75.48 \\
\hline San Ignacio & $190(20)$ & $14.37 \pm 13.98$ & 97.29 \\
\hline Totales & $504(93)$ & $9.51 \pm 10.04$ & 105.56 \\
\hline \multicolumn{4}{|l|}{ Plántulas } \\
\hline Normandía & $117(63)$ & $9.13 \pm 9.41$ & 103.02 \\
\hline La Piedra & 214 (74) & $9.59 \pm 7.04$ & 73.38 \\
\hline San Ignacio & $84(48)$ & $14.28 \pm 10.05$ & 70.40 \\
\hline Totales & $415(185)$ & $10.65 \pm 8.94$ & 83.95 \\
\hline
\end{tabular}



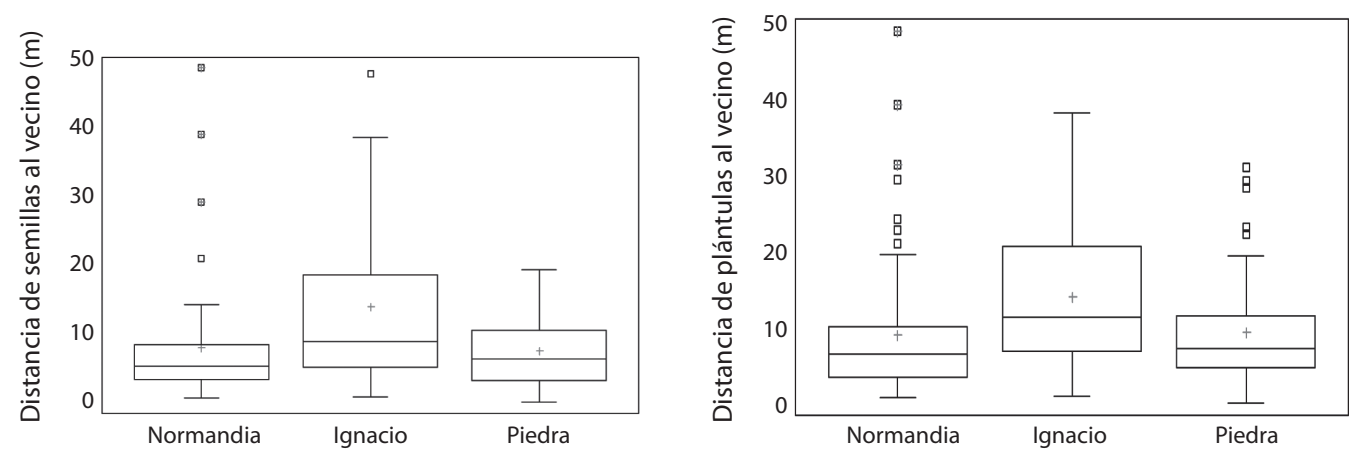

Fig. 3. Diagrama de cajas de las distancias de semillas y plántulas de $O$. bataua al vecino reproductivo más cercano en 100 parcelas de $1 \mathrm{~m}^{2}$ en cada uno de los fragmentos a). Distancia de semillas. b). Distancia de plántulas.

Fig. 3. Box plot distances of seeds and seedlings of $O$. bataua to the reproductive nearest neighbor in 100 plots of $1 \mathrm{~m}^{2}$ in each of the fragments a). Distance of seeds b) Distance of seedlings.

No se registraron diferencias entre las medias de las distancias de las semillas al vecino reproductivo más cercano (Kruskall-Wallis, $\mathrm{p}>0.05$ ) entre los tres fragmentos muestreados (Fig. 3a). Las plántulas sí mostraron diferencias significativas en las distancias entre los fragmentos (Kruskall-Wallis, $\mathrm{p}<0.05$ ). Las distancia de plántulas al vecino reproductivo más cercano estuvieron entre $0-50 \mathrm{~m}$, pero la mayoría sólo llegaron a $14 \mathrm{~m}$. Valores extremos en la distancia de plántulas al vecino reproductivo evidencian éxito en el establecimiento de semillas dispersadas por frugívoros (Fig. 3b).

\section{DISCUSIÓN}

La remoción de frutos de $O$. bataua fue alta y el consumo tanto en el racimo como en el suelo también lo fueron. Algunos frugívoros consumen los frutos cerca de la palma, lo que podría explicar el gregarismo de las plántulas $\mathrm{y}$ de los estadios juveniles (Rojas-Robles et al. 2008). Sin embargo, los frugívoros también alejaron las semillas y frutos a mayores distancias del árbol parental, los taparon, enterraron y en algunos casos los llevaron a sus cuevas.

$\mathrm{Al}$ cortar las raquilas, las ardillas se encargan de la dispersión primaria y en muchos casos, dejan disponibles frutos enteros para frugívoros que forrajean en el suelo y pueden actuar como dispersores secundarios. Si las ardillas no cortaran las raquilas ni las dejaran caer al suelo, los frutos se secarían en el racimo y no tendrían posibilidades de ser dispersados, pues éstos sólo caen cuando se secan. Por ello, las ardillas cumplen una función importante en la dispersión primaria de los frutos, al contrario de lo planteado por Sist (1989), quien sugiere que las ardillas consumen los frutos, pero no cumplen ningún papel en la dispersión. La función de las ardillas de dejar caer frutos disponibles para dispersores secundarios, en bosques más conservados lo pueden cumplir las aves grandes o primates (obs. pers.).

Aunque los mamíferos pueden actuar como depredadores de semillas, existen importantes beneficios de la interacción de las palmas con este grupo de consumidores, por ejemplo el alejamiento de las semillas del árbol parental (Forget 1991, Silva \& Tabarelli 2001, Galetti et al. 2006, Almeida \& Galetti 2007) y posiblemente la colonización de nuevas áreas dentro de los fragmentos estudiados. En los experimentos de dispersión de O. bataua, las semillas fueron transportadas hasta $53 \mathrm{~m}$ por frugívoros y se observó a una ardilla ( $S$. granatensis) transportar un pedazo de raquila con frutos maduros unos $62 \mathrm{~m}$ para almacenarlos. 
Estos eventos poco comunes o tal vez difíciles de observar, pueden ser de gran importancia para la semilla, si ésta es llevada a un microhábitat óptimo para la germinación, establecimiento de las plántulas y el futuro desarrollo de los juveniles. Los mamíferos pueden contribuir con la dispersión secundaria de semillas en buen estado y al remover el mesocarpo pueden ayudar a la semilla a disminuir el tiempo de germinación (obs. pers.).

A pesar de los porcentajes bajos de enterramiento de semillas a un centímetro de profundidad, éstos son importantes, ya que roedores que remueven o entierran y tapan semillas en sitios más favorables y con mejores condiciones microclimáticas para la germinación, ayudan a prevenir la depredación y el ataque tanto por vertebrados como por invertebrados, posiblemente aumentando la probabilidad de germinación de semillas y supervivencia de plántulas, convirtiéndose en dispersores efectivos de las poblaciones de O. bataua en el área de estudio. Sin embargo, hay que tener en cuenta la profundidad de enterramiento (Andresen \& Feer 2005), ya que la supervivencia de las semillas puede aumentar si es mayor la profundidad a la que fueron enterradas, pero la probabilidad de emergencia de las plántulas disminuye con la profundidad (Estrada \& Coates-Estrada 1991, Andresen \& Levey 2004). Las ventajas del enterramiento por roedores del género Dasyprocta para la germinación han sido ampliamente documentadas (Smythe 1970, 1989, Forget 1990, Silva \& Tabarelli 2001). Aunque Dasyprocta es considerado dispersor y depredador, en el caso de O. bataua, dado el tamaño y la dureza de la semilla, no vale la pena destruirla, si la parte rica en grasa esta en el mesocarpo blando.

Un dispersor efectivo debe remover el pericarpio de la semilla y depositarlo en una condición viable y en un sitio sostenible para la germinación y el establecimiento de las plántulas (Zona \& Henderson 1989). En el caso de $O$. bataua y para los frugívoros encontrados en este estudio (Sciurus granatensis, Microsciurus mimulus, Proechimys sp., Dasyprocta punctata y Marmosa robinsoni), podemos decir que estos remueven el pericarpio y depositan semillas en condiciones viables.

El depositar la semilla en un sitio sostenible para la germinación puede depender de la distancia. En este estudio el $22 \%$ de las semillas fueron dispersadas y alejadas distancias relativamente cortas (en promedio $3.1 \mathrm{~m}$ ), con menor número de registros a mayores distancias $(>50 \mathrm{~m})$. Sin embargo, estas distancias pueden estar subestimadas, pues en varias ocasiones los hilos de los frutos se enredaron evitando que los frugívoros los llevaran más lejos. Utilizando técnicas moleculares (Probabilidad de Identidad Materna), Karubian et al. (2010) en Bilsa, Chocó biogeográfico ecuatoriano, en un bosque más conservado encontraron que las semillas de O. bataua se mueven un promedio de $375 \mathrm{~m}$ y que hay una alta heterogeneidad genética de las semillas en parcelas en las cuales se realiza el lek del pájaro paraguas Cephalopterus penduliger. A futuro realizaremos este mismo tipo de análisis genético para Porce y comparar con Bilsa y con un tercer sitio más conservado en la Orinoquía colombiana, las distancias de dispersión y la heterogeneidad genética o posibles cuellos de botella que puedan observarse en Porce como sitio más fragmentado y defaunado.

Las condiciones del micro-sitio al cual los frugívoros llevan las semillas son fundamentales para determinar la efectividad de la dispersión y esta nueva superficie es muy variable dependiendo del comportamiento del dispersor. Esto también fue comprobado por Karubian et al. (2010) para C. penduliger, ave frugívora cuyo sistema de apareamiento de lek crea un patrón específico de dispersión de semillas de larga distancia, que puede distinguirse de los demás procesos de dispersión, afectando así el movimiento de genes y la estructura genética de $O$. bataua, uno de sus alimentos preferidos.

Que la semilla sea alejada de la planta madre es una condición necesaria, aunque no suficiente, para que el proceso de dispersión sea exitoso (Herrera 2001). En este estudio el $6 \%$ de semillas y frutos fueron enterrados, tapados y llevados a cuevas y en esta nueva superficie las semillas podrían tener mayor 
éxito en la germinación y el establecimiento. En este caso no tenemos datos de germinación de las semillas dispersadas en los experimentos de remoción. Sin embargo, en las parcelas de un metro cuadrado se encontraron semillas y plántulas a distancias de hasta $50 \mathrm{~m}$ del vecino reproductivo más cercano, evidenciando eventos de dispersión por fauna y éxito en la germinación y el establecimiento de las plántulas a diferentes distancias, corroborando con ello en parte, la eficiencia de los dispersores de $O$. bataua en el área de estudio.

En la Guayana Francesa, los principales consumidores de frutos de $O$. bataua registrados fueron las aves Pionus fuscus, Pionites melanocephala, Amazona ochrocephala y A. farinosa (todos Psittacidae), también ardillas y primates (Sist 1989). En la región de la Amazonía, Kanh \& De Granville (1992) registraron aves grandes como: A. farinosa, A. ochrocephala, Penelope marail, Perissocephalus tricolor, Pionites melanocephala, Pionus fuscus y Ramphastos tucanus, la ardilla Sciurus aestuans y los primates Ateles paniscus y Cebus nigrivitatus, consumiendo frutos de $O$. bataua. Otras especies registradas comiendo frutos de O. bataua son Pecari tajacu, Tayassu pecari, Steatornis caripensis, Pitecia monachus, Cebus albifrons, Ateles belzebuth, Ara macao y Pipile cumanensis. Aunque estos registros son más de carácter puntual que sistemáticos, abarcan 14 especies de aves y 13 especies de mamíferos consumiendo los frutos de $O$. bataua (Sist 1989, Zona \& Herderson 1989, Bodmer 1991, Kahn \& Granville 1992, Peres 1994, Stevenson et al. 2000), indicando que el gremio de frugívoros de esta palma en bosques primarios y continuos es mucho más amplio que el encontrado en nuestra área de estudio, en la cual, en un año y medio con diferentes métodos de muestreo, sólo se registró una especie de ave y cinco especies de mamíferos.

Los bosques donde se realizó este estudio han sido fragmentados para dar paso a la ganadería (Agudelo \& Restrepo 2004), intervenidos y sometidos a cacería (observ. pers), lo cual ha llevado a la disminución y en algunos casos extinción local de poblaciones de las especies de mamíferos y aves grandes que son las que en otras regiones procuran más los frutos de $O$. bataua.

Una gran diversidad de aves y mamíferos de medio y gran porte (mayores a dos kilos), que están entre los animales más cazados, consumen, dispersan y depredan semillas afectando el reclutamiento en las poblaciones de plántulas (Wright \& Duber 2001, Wright 2003). Plantas con frutos y semillas grandes y bien protegidas como las de O. bataua, son las más afectadas por la fragmentación, pues necesitan de frugívoros grandes para la dispersión de sus semillas (Pizo 2004). Algunos estudios han demostrado que palmas como Attalea sp. y Astrocaryum sp. tienen menor dispersión en áreas con alta frecuencia de cacería (Wright 2003). Además los frugívoros grandes son los más susceptibles a la fragmentación de su hábitat, pues están entre las presas preferidas por los cazadores (Redford 1992, Peres 2000) y no encuentran en los fragmentos, todos los recursos necesarios para sobrevivir a lo largo del año. Como consecuencia de la ausencia o baja abundancia de animales frugívoros en los fragmentos, el éxito reproductivo de las plantas, medido por la remoción de sus frutos, puede ser drásticamente afectado (Galetti et al. 2003).

$\mathrm{Al}$ desaparecer los depredadores por fragmentación, cacería o modificaciones del hábitat, aumenta el tamaño poblacional de especies de roedores y granívoros pequeños (Jordano et al. 2006). Ardillas, marsupiales, pequeños roedores y pequeñas aves granívoras generalmente no son cazados, pudiendo aumentar sus poblaciones considerablemente en áreas defaunadas, como resultado de la falta de competidores y depredadores (Pizo \& Vieira 2004). Este puede ser el caso de las ardillas en nuestra área de estudio. A pesar de no tener datos sobre abundancias de ardillas era muy común observarlas y escucharlas. Adicionalmente fueron las especies más frecuentes en los focales y las únicas en los registros fotográficos en los racimos, dando cuenta de su abundancia. De esta forma, las ardillas pueden ejercer gran presión por el recurso frutos, eliminando otros frugívoros competidores. Quizás por ello, a pesar 
de quedar en los fragmentos estudiados algunas aves como: Ortalis columbiana, Aburria aburri y tucanes como Pteroglossus torquatus, Ramphastos citreolaemus y Ramphastos swainsonii que podrían consumir los frutos de $O$. bataua, nunca fueron observadas y por el contario prefirieron visitar otras especies de árboles con frutos carnosos ornitócoros, pero no los racimos de O. bataua, al menos durante el tiempo de observación.

Los 751 frutos y semillas colocados en los experimentos de dispersión fueron pelados y roídos, pero ninguno fue partido o dañado, de tal forma que los frugívoros consumen el mesocarpo rico en grasas, proteínas y carbohidratos (Balick 1992) y descartan semillas sanas que podrían germinar. No ocurre lo mismo con la palma Syagrus romanzoffiana en fragmentos de Mata Atlántica brasileña de diferentes tamaños, donde la ardilla Sciurus ingrami es un importante depredador de sus semillas. No hay sitio seguro para el establecimiento de esta palma en fragmentos medianos y pequeños. En fragmentos grandes la supervivencia de las semillas estuvo relacionada con menor abundancia de ardillas y el mantenimiento de las condiciones en el interior del bosque, en contraste con las condiciones en el borde y en los claros (Fleury \& Galetti 2006).

En $O$. bataua la abundante producción de frutos (Rojas \& Stiles 2009), el tamaño, peso, la intensa remoción de frutos del racimo, las cortas distancias de dispersión por frugívoros y posiblemente la ausencia de frugívoros de gran porte encargados de la dispersión de más larga distancia, generan una sombra de semillas espacialmente restringida responsable de los patrones de distribución agregados en plántulas $\mathrm{y}$ posteriormente en juveniles y sub-adultos (Rojas et al. 2008).

El alto porcentaje y el corto tiempo de germinación de semillas, así como la robustez de semillas y plántulas, expresada en una relativa baja mortalidad (Rojas et al. 2008), nos lleva a pensar que $O$. bataua posee capacidad para sobrevivir en agregaciones densas. A pesar de las limitaciones en la dispersión, la alta supervivencia hace que sea una de las especies de palmas más abundantes y exclusivas de bosques secundarios viejos y bosques primarios en el área de estudio como lo plantean Yepes et al. (2010).

A lo largo de su ámbito de distribución, las poblaciones de $O$. bataua han sido reducidas por su explotación intensiva y por la trasformación o reducción de su hábitat natural y ello ha alterado procesos ecológicos que son fundamentales para el mantenimiento de las poblaciones de la especie. La sobre explotación de los frutos o de los estadios juveniles de esta palma, como fue observado en el área de estudio, puede afectar la estructura de edades, la dinámica poblaciones y su potencial de extracción futura. Estudios de más largo plazo sobre demografía, regeneración, reproducción, cambios en los procesos e interacciones ecológicas por las trasformaciones del hábitat, fragmentación, defaunación, etc., son de vital importancia. Sin embargo, el manejo y la explotación de esta palma en el futuro dependerán en gran medida de la conservación de los hábitats de bosque donde ella es aún un elemento dominante.

\section{AGRADECIMIENTOS}

A la División de Investigaciones de la Universidad Nacional de Colombia, sede Bogotá (DIB) por la financiación del proyecto; al Departamento de Biología de la misma universidad, a Empresas Públicas de Medellín (EPM) por el apoyo logístico, a los auxiliares de campo, Enrique Gutiérrez, Cesar Cuervo, Jaime Bello y Luis Núñez. A Sergio Solari por la identificación de los frugívoros fotografiados.

\section{RESUMEN}

En zonas bajas tropicales, en bosques continuos y relativamente poco intervenidos, los frutos de la palma Oenocarpus bataua Mart., son consumidos por diversas especies de vertebrados, sin embargo, no hay estudios de dispersión de más largo plazo con esta especie. Entre agosto 2005-septiembre 2006 se realizaron observaciones de remoción de frutos, conteo de frutos comidos, observaciones focales, registros de frugivoría mediante trampas fotográficas, experimentos de dispersión y parcelas para 
determinar distribución espacial de Oenocarpus bataua. Cinco especies de mamíferos comen, dispersan, entierran (Sciurus granatensis, Microsciurus mimulus, Dasyprocta punctata y Proechimys sp.), tapan (Marmosa robinsoni) y llevan a las cuevas (Marmosa robinsoni y Proechimys sp.), los frutos de O. bataua, sin dañar la semilla. El $21.7 \%$ de los frutos fueron dispersados, $13.2 \%$ roídos o pelados, $5.6 \%$ tapados, enterrados y llevados a cuevas. La distancia media de remoción de semillas y frutos fue $3.1 \mathrm{~m}$, aunque en menor proporción se registraron eventos de dispersión $>$ a 50m. La abundante producción, el tamaño, el peso, la intensa remoción de los frutos por frugívoros, las cortas distancias de dispersión, la ausencia de frugívoros de gran porte disminuidos por cacería y fragmentación, encargados de la dispersión de larga distancia y el aumento de roedores, especialmente ardillas que presionan fuertemente el recurso frutos, generan una lluvia de semillas espacialmente restringida, posiblemente responsable de los patrones de distribución agregados en semillas, plántulas y posteriormente en juveniles y sub-adultos.

Palabras clave: Oenocarpus bataua, palmas, frugivoría, cámaras trampa, distribución espacial, fragmentación, defaunación, Colombia.

\section{REFERENCIAS}

Agudelo, A. \& J.C. Restrepo. 2004. Análisis multitemporal de coberturas vegetales en un bosque húmedo tropical (Río Porce, Colombia). Tesis de Maestría, Universidad Nacional de Colombia, Medellin, Colombia.

Almeida, L.B. \& M. Galetti. 2007. Seed dispersal and spatial distribution of Attalea geraensis (Arecaceae) in two remnants of Cerrado in Southeastern Brazil. Acta. Oecol. 32: 180-187.

Andresen, E.D. \& J. Levey. 2004. Effects of dung and seed size on secondary dispersal, seed predation, and seedling establishment of rain forest trees. Oecologia 139: 145-54.

Andresen, E. \& F. Feer. 2005. The role of dung beetles as secondary seed dispersers and their effect on plant regeneration in tropical rainforests, p. 331-349. In P.M. Forget, J. Lambert, P. Hulme \& S.B. Vander Wall (eds.). Seed Fate: predation, dispersal and seedling establishment. CAB International, Oxofordshire, Reino Unido.

Balick, M.J. 1992. Jessenia y Oenocarpus: Palmas aceiteras neotropicales dignas de ser domesticadas. FAO, Roma, Italia.

Barot, S., J. Gignoux \& J.C. Menaut 1999. Population structure and life cycle of Borassus aethiopum Mart.: Evidence of early senescence in a palm tree. Biotropica 3: 439-448.
Bodmer, R.E. 1991. Strategies of seed dispersal and seed predation in Amazonian ungulates. Biotropica 23: 255-261.

Borchsenius, F., H. Borgtoft-Pedersen \& H. Balslev. 1998. Manual to the palms of Ecuador. AAU Report 37: 1-240. Department of Systematic Botany, Universidad of Aarhus, Dinamarca.

Connell, J.H. 1971. On the role of natural enemies in preventing competitive exclusion in some marine animals y in rain forest trees, p. 298-312. In P.J. Den Boer \& G.R. Gradwell (eds.). Dynamics of Populations. Netherlys, Pudoc, Wageningen, Países Bajos.

Cordeiro, N.J. \& H.F. Howe. 2003. Forest fragmentation severs mutualism between seed dispersers and an endemic African tree. Proc. Natl. Acad. Sci. USA. 100: 14052-4056.

Corporación Ambiental. 2001. Plan de monitoreo y seguimiento de la componente físico-biótica en la zona del proyecto hidroeléctrico Porce II. Ejecución del Segundo Monitoreo de Fauna. Universidad de Antioquía, Medellín, Colombia.

Dirzo, R. \& A. Miranda. 1990. Contemporary Neotropical defaunation and forest structure, function, and diverity- A sequel to John Terborgh. Conservat. Biol. 4: 444-447.

Emmons, L. \& F. Feer. 1999. Mamíferos de los bosques húmedos de América Topical. FAN, Santa Cruz, Bolivia.

Estrada, A. \& R. Coates-Estrada. 1991. Howler monkeys (Alouatta palliata) dung Beetles (Scarabaeidae) and seed dispersal: ecological interactions in the tropical rain Forest of Los Tuxtla, Mexico. J. Trop. Ecol. 7: 459-574.

Fleury, M. \& M. Galetti. 2006. Forest fragment size and microhabitat effects on palm seed predation. Biol. Conservat. 131: 1-13.

Forget, P.M. 1990. Seed-dispersal of Vouacapoua americana (Caesalpiniaceae) by caviomorph rodents in French Guiana. J. Trop. Ecol. 6: 459-468.

Forget, P.M. 1991. Scatterhoarding of Astrocaryum paramaca by Proechimys in French Guiana: comparison whit Myoprocta exilis. Trop. Ecol. 32: 155-167.

Forget, P.M. \& D.G. Wenny. 2005. How to elucidate seed fate? A review of methods used to study seed removal and secundary seed dispersal, p. 379-393. In P.M. Forget, J.E. Lambert, P.E. Hulme \& B. Vander Wall (eds.). Seed fate. Predation, dispersal and seedling establishment. CABI, Londres, Inglaterra.

Galeano, G. \& R. Bernal. 1987. Palmas del departamento de Antioquia: Región Occidental. Universidad Nacional de Colombia, Bogotá, Colombia.

Galetti, M., C. Costa \& E. Cazetta 2003. Effects of forest fragmentation, anthropogenic edges and fruit color 
on the consumption of ornithochoric fruits. Biol. Conservat. 111: 269-273.

Galetti, M., C.I. Donatti, A.S. Pires, P. Jr. Guimarães \& P. Jordan. 2006. Seed survival and dispersal of an endemic Atlantic forest palm: the combined effects of defaunation and forest fragmentation. Bot. J. Linn. Soc. 151: 141-149.

Hamilton, W.D. \& R. May. 1977. Dispersal in stable habitats. Nature 269: 578-581.

Henderson, A., G. Galeano \& R. Bernal 1995. Field guide to the palms of the Americas. Princeton, Nueva Jersey, EEUU.

Henderson, A. 2002. Evolution and ecology of palms. The New York Botanical Garden, Nueva York, EEUU.

Herrera, C.M. 2001. Dispersión de semillas por animales en el Mediterráneo: ecología y evolución, p. 125-152. In R. Zamora \& F.I. Pugnaire (eds.). Ecosistemas Mediterráneos: análisis funcional. Colección Textos Universitarios, Madrid, España.

Holdridge, L. 1996. Ecología basada en zonas de vida. Instituto Interamericano de Cooperación para la Agricultura, San José, Costa Rica.

Hollander, J.L \& S.B. Vander Wall. 2004. Effectiveness of six species of rodents as dispersers of single leaf piñon pine (Pinus monophylla). Oecologia 138:57-65.

Howe, H.F. 1977. Bird activity y seed dispersal of a tropical wet forest tree. Ecology 58:539-550.

Howe, H.F. \& J. Smallwood. 1982. Ecology of seed dispersal. Annu. Rev. Ecol. Syst. 13: 201-228.

Howe, H.F. 1990. Seed dispersal by birds and mammals: implications for seedling demography, p. 191-218. In K.S. Bawa \& M. Hadle (eds.). Man and the Biosphere, Reproductive Ecology of Tropical Forest Plant, UNESCO, Paris, Francia.

Janzen, D.H. 1970. Herbivores and the number of species in tropical forest. Am. Nat. 107: 501-528.

Jordano, P. 2000. Fruits and frugivory, p. 125-166. In M. Fenner (eds.). Seeds: the ecology of regeneration in plant communities. CAB, Wallingford, Reino Unido.

Jordano, P. \& E.W. Schupp. 2000. Seed disperser effectiveness: the quantity component and patterns of seed rain for Prunus mahaleb. Ecol. Monogr. 70:591-615.

Jordano, P. \& J.A. Godoy. 2002. Frugivore-regenerated Seed Shadows: a landscape view of demographic and genetic effects, p. 305-321. In D.J. Levey, W.R. Silva \& M. Galetti (eds.). Seed dispersal and frugivory: ecology, evolution and conservation. CAB International, Reino Unido.

Jordano, P., M. Galetti, M.A. Pizo \& W.E. Silva. 2006. Ligando Frugivoria e Dispersão de Sementes à Biologia da Conservação, p. 411-436. In C.F. Duarte, H.G. Bergallo, M.A. Dos Santos, A.E.Va (eds.). Biologia da conservação: essências. Rima, São Paulo, Brasil.
Karubian, J., V. Sork, T. Roorda, R. Duraes \& S. Thomas, 2010. Destination-based seed dispersal homogenizes genetic structure of a tropical palm. Mol. Ecol. 19: 1745-1753.

Kahn, F. \& J.J. De Granville. 1992. Palms in forest ecosystems of Amazonia. Springer Verlag, Berlin, Alemania.

Nathan, R. \& C. Muller-Landau. 2000. Spatial patterns of seed dispersal, their determinants and consequences for recruitment. Trends. Ecol. Evol. 15: 278-285.

Peres, C.A. 1994. Composition, density and fruiting phenology of arborescent palms in Amazonian terra firme forest. Biotropica 26: 285-294.

Peres, C.A. 2000. Effects of subsistence hunting on vertebrate community structure in Amazonian forests. Conservat. Biol. 14: 240-253.

Pizo, M.A. 2004. Frugivory and habitat use by fruit-eating birds in a fragmented landscape of southeast Brazil. Ornitologia Neotropical 15: 117-126.

Pizo, M.A. \& E.M. Vieira. 2004. Granivorous birds as important post-dispersal seed predators in a Brazilian forest fragment. Biotropica 36: 417-423.

Redford, K.H. 1992. The empty forest. Bioscience 42: 412-422.

Rojas-Robles, R. 2008. Fenología, frugivoría, dispersión de semillas y distribución espacial de la palma Oenocarpus bataua en tres fragmentos de bosque subandino de Colombia”. Tesis de Doctorado, Universidad Nacional de Colombia, Bogotá, Colombia.

Rojas-Robles, R., A. Correa \& E. Serna. 2008. Sombra de semillas, supervivencia de plántulas y distribución espacial de Oenocarpus bataua (Arecaceae) en un bosque de los Andes colombianos. Actual. Biol. 30: 137-150.

Rojas-Robles, R. \& F.G. Stiles. 2009. Analysis of a supraannual cycle: reproductive phenology of the palm Oenocarpus bataua, in a forest of the Colombian Andes. J.Trop. Ecol. 25: 41-51.

Silva, M.G. \& M. Tabarelli. 2001. Seed dispersal, plant recruitment and spatial distribution of Bactris acanthocarpa Martius (Arecaceae) in a remnant of Atlantic forest in northeast Brazil. Acta. Oecol. 22: 289-268.

Sist, P. 1989. Strategies de regeneration de quelques palmiers en foret Guyanaise. Tesis de Doctorado, Universidad de Paris, Francia.

Smythe, N. 1970. Relationships between fruiting seasons and seed dispersal methods in a neotropical forest. Am. Nat. 104: 25-35.

Smythe, N. 1989. Seed survival in the palm Astrocaryum standleyanum: evidencia for dependence upon its seed dispersers. Biotropica 21: 50-56. 
Snow, D.W. 1981. Tropical frugivorous birds and theirs food plants: A world survey. Biotropica 13: 1-14.

Stevenson, P.R., M.J. Quiñonez \& J.A. Ahumada. 2000. Influence of fruit availability on ecological overlap among four neotropical primates at Tinigua National park, Colombia. Biotropica 32: 533-544.

Vander Wall, S.B., P.M. Forget, J.E. Lambert \& P.E. Hulme. 2005. Seed fate pathways: filling the gap between parents and offspring, p. 1-8. In P.M Forget, J.M. Labert, P.E. Hulme \& S.B. Vander Wall (eds.). Seed Fate. Predation, dispersal and seedling establishment. CAB International, Reino Unido.

Wang, B.C. \& T.B. Smith. 2002. Closing the seed dispersal loop. Trends Ecol. Evol. 17: 379-385.
Wright, S.J. \& H.C. Duber. 2001. Poachers and forest fragmentation alter seed dispersal, seed survival, and seedling recruitment in the palm Attalea butyracea with implications for tropical tree diversity. Biotropica 33: 583-5595.

Wright, S.J. 2003. The myriad consequences of hunting for vertebrates and plants in tropical forests. Perspect. Plant. Ecol. Evol. Systemat. 6: 73-86.

Yepes, A.P., J.I. Del Valle, S.L. Jaramillo \& S.A. Orrego. Recuperación estructural en bosques sucesionales andinos de Porce (Antioquia, Colombia). Rev. Biol. Trop. 58: 427-445.

Zona, S. \& A. Henderson. 1989. A review of animalmediated seed dispersal of palms. Selbyana11: 6-21. 
